\title{
PREFERENTIAL OPPONENT SELECTION IN PUBLIC GOODS GAMES
}

\author{
MARKUS BREDE \\ School of Electronics and Computer Science, \\ University of Southampton, University Road, \\ Southampton, Hampshire SO17 1BJ, United Kingdom \\ Markus.Brede@soton.ac.uk
}

Received 5 March 2012

Revised 14 May 2012

Accepted 21 May 2012

Published 6 July 2012

\begin{abstract}
This paper discusses preferential opponent selection in public goods games. It is shown that a preference to play with successful opponents strongly enhances the prevalence of cooperation. The finding is robust on spatial grids and heterogeneous networks. Importantly, I also demonstrate that positive opponent selection biases can evolve and become dominant in initially randomly mixed populations without selection bias.
\end{abstract}

Keywords: Public goods games; evolutionary game theory; complex networks.

\section{Introduction}

Altruism describes individual behavior that benefits the group, but comes at a cost to the individual. In spite of this, altruism is found in many contexts in biology, economics and social science and understanding it still poses some major challenges. The key technique to understand the prevalence of altruism is the framework provided by evolutionary game theory [1]. It explores the evolution of strategies in populations of individuals in game theoretic settings.

The evolutionary prisoner's dilemma game [2] and evolutionary public goods games (PGGs) are probably the two most studied models that can illustrate the emergence of cooperation in competitive evolutionary games. Whereas the prisoner's dilemma game is a model for cooperation with bilateral interactions, a PGG is the standard model for cooperation in situations with multilateral interactions.

A typical PGG with binary strategy choices between $N$ agents can be described as follows. All agents simultaneously make a choice whether they contribute a fixed amount of $\$ 1$ to a public pool or not. After contributions have been collected, the public pool is multiplied by a factor $r>1$ and divided into equal shares which 
are handed back to all agents who took part in the game. In this setting agents who do not contribute ("defectors") can earn higher payoffs at the expense of agents who contributed ("cooperators") and thus be individually better off than the latter. However, the outcome for the group is maximized if all agents cooperate, since then all moneys earn the benefit of the public good expressed by a multiplier $r>1$. In an evolutionary setting with well-mixed populations defection always prevails for $r>1$ in such one-off games as described above.

Modeling the emergence of cooperation in evolutionary social dilemmas has attracted considerable interest over the past years and various mechanisms that can allow for the survival of cooperation have been uncovered. Some very wellknown ones amongst them are kin selection [3], the evolution of complex behavior in repeated games $[2,4]$, indirect reciprocity [5], volunteering $[6,7]$ and network reciprocity [8-16], see [17] for a review on the topic. Since it has been discovered that many complex biological and social systems are connected by complex networks (cf. [18-20] for reviews), the latter mechanism has shifted into the focus of interest. Recent studies have shown that heterogeneous interaction topologies that allow some agents to earn much larger payoffs than others strongly boost cooperation [1216]. Other models have explored other sources of heterogeneity - like payoff matrix noise [21] or quenched stochasticity in the payoff matrix $[22,23]$, heterogeneity in strategy adoption [24], wealth accumulation [25] or the Matthew effect [26] — for agents interacting on regular networks. In most of these settings a similar boost of cooperation can be observed.

Some other relevant recent studies have explored the role of "aspirations" in the prisoner's dilemma game [27-29] and in PGGs [30, 31]. These models interpret "aspirations" as a bias in the selection of reference agents for strategy adoption. An important result of this work is that a tendency of agents for preferentially imitating very successful agents supports cooperation. Similarly, [32] has investigated biases in opponent selection in the context of the prisoner's dilemma, finding that a preference to play against successful agents can give a strong advantage to cooperation. This paper follows up on the latter study, exploring the mechanism of opponent selection in the context of the PGG. Similar to [32], it is found that opponent selection biases can strongly enhance cooperation far beyond the effect of network reciprocity alone.

It is worthwhile noting that opponent selection has also been addressed in coevolutionary models of cooperation in the prisoner's dilemma, in which, subject to certain rules, agents rewire (or reweigh) connections during the evolution of strategies [33-40], see [41] for a recent review. The present framework is different from this work in two regards: (i) by the opponent selection mechanism effectively a dynamical weighing of links is applied, but there is no positive feedback to explicitely reinforce links that generate larger than average payoffs as e.g., in [40] and (ii) as regards strategy diffusion the opponent selection mechanism leaves the network topology unaltered. 
This paper is organized as follows. Section 2 introduces the details of the evolutionary PGG game and describes the model of opponent selection. Section 3.1 then explores the evolutionary PGG in space and Sec. 3.2 extends these results to heterogeneous networks. In Sec. 3.3 it is explored how opponent selection biases can arise by evolution from an initially unskewed population. Section 4 which summarizes and discusses the presented results concludes the paper.

\section{Model}

Following the standard model in the literature on games on networks agents are identified with nodes and interactions are represented by the links of a network [8-16]. The network is considered as binary and undirected, given by its adjacency matrix $A=\left(a_{i j}\right)_{i, j=1}^{N}$. One sets $a_{i j}=1$ if the agents $i$ and $j$ are linked and $a_{i j}=0$ otherwise. Agents interact with their network neighbors in two ways. First, if an agent is selected for game play, a PGG is played involving the selected agent and all of its neighbors. Second, the same links that define interactions for game play also define sets of possible partners for strategy spread.

More specifically, I consider a set of $N$ agents with pure strategies, either to contribute to all PGG they play (strategy $s=1$, "cooperate") or not to contribute to all PGGs (strategy $s=0$, "defect"). A round of game play consists of every agent selecting a game partner. Game partners are selected according to previous success. The selection rule is probabilistic and an agent $i$ will select one of its neighbors $j$ for game play with probability

$$
P_{\text {select }}(i, j)=\frac{\exp \left(w\left(\pi_{j}-\pi_{i}\right)\right)}{\sum_{j} a_{i j} \exp \left(w\left(\pi_{j}-\pi_{i}\right)\right)},
$$

where $\pi_{i}$ stands for the accumulated payoff of agent $i$ in the previous round of game play. The parameter $w$ characterizes the agents' biases towards selecting more or less successful partners for game play. A choice of $w>0$ gives a preference for more successful partners and a choice of $w<0$ biases selection towards selecting less successful opponents. Choosing $w=0$ reproduces the unbiased game, albeit at a different ratio of timescales of game play and strategy spread than in the usual deterministic PGG in which PGGs are played between a focal agent and all of its neighbors before strategy spread occurs. This issue has recently been discussed in the context of the prisoner's dilemma game on scale-free networks [42]. For the framework discussed here, the same timescales as in the deterministic game could be reproduced by repeating the selection procedure and game play as many times as an agent has neighbors. Modifying the game setup in this way does not change the qualitative result. For reasons of simplicity (and to save computation time) this study will focus on the game where every agent selects one game partner in each round of game play.

If agent $j$ is selected for game play, a PGG involving agent $j$ and all of its neighbors will take place. As in a standard PGG with binary strategy choices, 
payoffs from a game centered around $j$ are

$$
\pi(s)= \begin{cases}\frac{r\left(s_{j}+\sum_{i} a_{j i} s_{i}\right)}{1+\sum_{i} a_{j i}}-1 & \text { if } s=1 \\ \frac{r\left(s_{j}+\sum_{i} a_{j i} s_{i}\right)}{1+\sum_{i} a_{j i}} & \text { if } s=0,\end{cases}
$$

i.e., a cooperator receives the benefits of the game minus his contribution and defectors receive the benefits of the game without deduction (as they contributed nothing). The parameter $r>1$ quantifies the severity of the dilemma situation, the smaller $r$ the "tougher" the public goods dilemma.

After cumulated payoffs have been calculated strategies can adapt in the population. Strategy adaptation is modeled as a synchronous process using Fermipairwise updating as in [43], i.e., the focus agent, say $i$, selects one neighbor at random for reference and imitates the neighbors strategy with probability

$$
P\left(s_{i} \rightarrow s_{j}\right)=\frac{1}{1+\exp \left(\left(\pi_{i}-\pi_{j}\right) / \kappa\right)},
$$

where the parameter $\kappa$ is a measure for the noise in the updating process.

Initializing the setup with random allocations of $50 \%$ cooperator and $50 \%$ defector agents and zero payoffs, game play and strategy updating are iterated till a quasi-stationary state has been reached. Following this, averages of the fraction of cooperators $n_{c}$ are calculated over a further $10^{4}$ iterations. For spatial networks, simulations have been carried out on lattices of sizes between $100 \times 100$ upto $400 \times 400$, for scale-free networks $N=10^{4}$ nodes are simulated. In case of experiments on heterogeneous networks the stationary fractions of cooperators are further averaged over at least 500 network realizations.

\section{Results}

\subsection{Evolution of cooperation in spatial games}

Figure 1 gives the dependence of the average stationary fraction of cooperators on the order parameter $r$ for several game partner selection biases $w$ in spatial public goods games. For tough dilemma settings and low values of the order parameter $r$ defection is dominant. Increasing $r$ at some stage cooperators can coexist with defectors until cooperation dominates for very large $r$. Figure 1 illustrates that opponent selection biases have a strong impact on the transition from defection to coexistence of defection and cooperation and finally dominance of cooperation. They effect both the critical value of $r$ at which cooperators go extinct, but also the general shape of the transition.

Analyzing the data in Fig. 1, one immediately notes that negative biases ("playing against less successful opponents") make it substantially harder for cooperation to emerge. Positive biases ("playing against more successful opponents") 


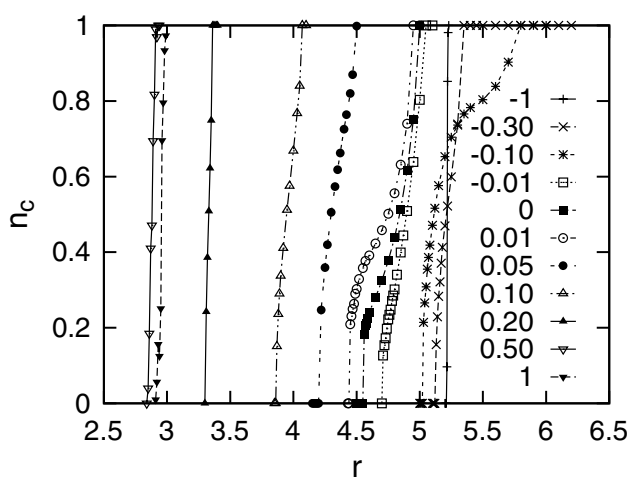

Fig. 1. Dependence of the stationary fraction of cooperators on the factor $r$ for the spatial public goods game with opponent selection biases (see legend for values of $w$ ) for $\kappa=0.1$. The data have been obtained on a $100 \times 100$ grid with von Neumann neighborhoods.

typically have the opposite effect: Cooperation can emerge for tougher dilemma settings then in the other case. An exception to this rule are very strong positive selection biases at which the prevalence of cooperation declines slightly. This effect will be discussed in more detail below.

A better overview about the the effect of selection biases on cooperation can be obtained from phase diagrams that show the dependence of the onset of cooperation $r_{c}$ on the selection bias parameter $w$. This diagram is given in Fig. 2 for low, intermediate and large noise levels $\kappa$ in the strategy adaptation process. The general rule that positive opponent selection biases favor cooperation holds independent of the noise in the strategy adaption. For all considered noise levels there is a steep transition between the parameter range of $w<0$ and $w>0$ and $r_{c}$ is found to converge quickly in the limits $w \rightarrow \pm \infty$.

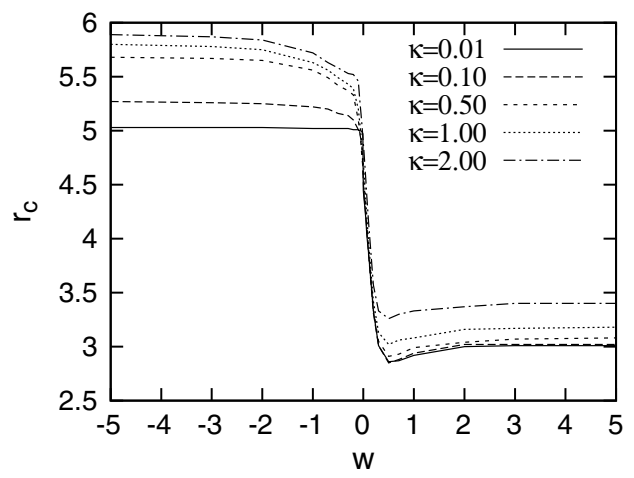

Fig. 2. Dependendence of the threshold value of the parameter $r$ at which cooperators go extinct on the preference parameter $w$ for various noise levels $\kappa$. Above the respective lines cooperators can survive (or even dominate), below defection is the only viable strategy. Best conditions for cooperation can be achieved for $\kappa=0.01$ and $w=0.5$ for which $r_{c}=2.85 \pm 0.01$. 
How can the strong boost for cooperation from positive opponent selection biases be explained? The argument runs along similar lines as for the analog analysis for cases of the prisoner's dilemma game advanced in [32]. In a structured population cooperation can survive by forming close-knit pockets of cooperators [8, 11]. Such an arrangement protects cooperators inside the clusters of cooperation from invasions by defectors and benefits cooperators at the fringe of the cluster by providing them with opportunities to generate payoff from cooperative interactions with other cooperators. Once pockets of cooperators have been established, positive opponent selection biases will reinforce the mutual support of cooperators. This is so, because opponent selection biases reduce interactions with defectors (which earn less payoff than cooperators inside the pockets of cooperation) and increase interactions between cooperators at the fringe and inside clusters of cooperators. In this way a hierarchy of payoffs is established: Nodes inside clusters of cooperators have the highest payoffs, because they (i) only interact with other cooperators and (ii) attract additional interactions in comparison to other agents with less payoff. Opponent selection biases thus shield clusters of cooperators.

This protective shielding effect is absent in the initially well-mixed populations. In well-mixed populations, defectors can generally obtain larger payoffs than cooperators. Consequently, positive opponent selection biases increase the (relative) number of interactions between cooperators and defectors and negative selection biases reduce the number of interactions between cooperators and defectors, i.e., help cooperators to avoid defectors. Hence, starting from a random initial allocation of strategies, strong positive opponent selection biases can lead to the extinction of cooperation before a structured arrangement of cooperators and defectors has been reached.

The result of these two opposing tendencies — strong support for cooperation from positive biases in structured arrangements and a strong boost for defection from positive biases in randomly mixed populations - is an optimal preference level $w$ for opponent selection. This is seen as a shallow minimum in $r_{c}$ for $w \approx$ $0.3, \ldots, 0.5$ (the details depending on the noise level $\kappa$ ) which marks a region in parameter space where cooperation is maximized, cf. Fig. 2.

The above numerical data are complemented by the data exhibited in Fig. 3, which illustrates the full phase diagrams, demarcating regions where defectors or cooperators dominate from the coexistence region in between. The data are given for low $\kappa=0.1$ (panel a), intermediate $\kappa=0.5$ (panel b) and large $\kappa=2.0$ (panel c) noise levels in the strategy adaptation process. The figures illustrate changes in the shape of the transition between cooperation and defection with changes in opponent selection biases. There are several interesting observations to be made.

First, for all noise levels transitions become very steep for large positive selection biases, i.e., the coexistence region almost vanishes for $w>0.5$. Large negative biases also reduce the size of the coexistence region, but the effect is much more gradual, in particular if $\kappa$ is large. The size of the coexistence region is maximized for slightly negative selection biases around $w=-0.3$ - a region in which negative 


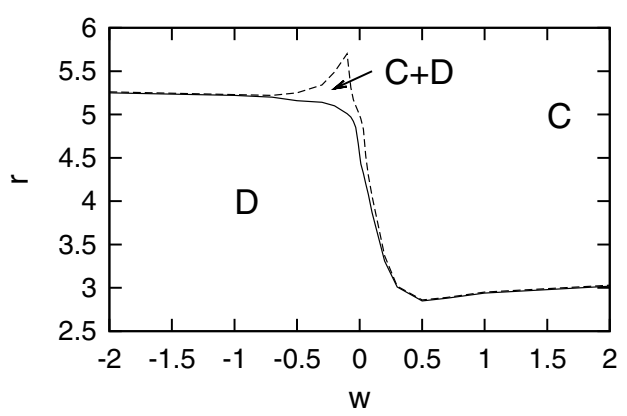

(a)

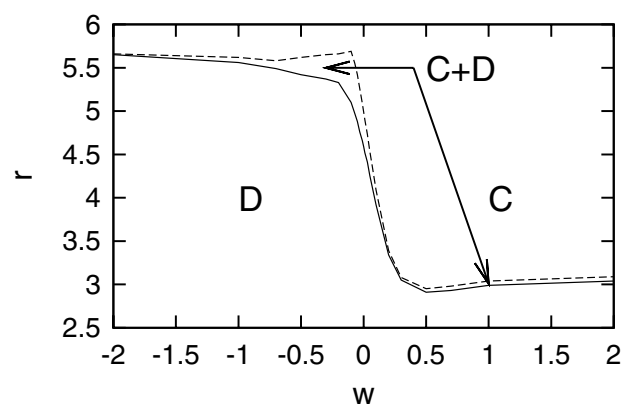

(b)

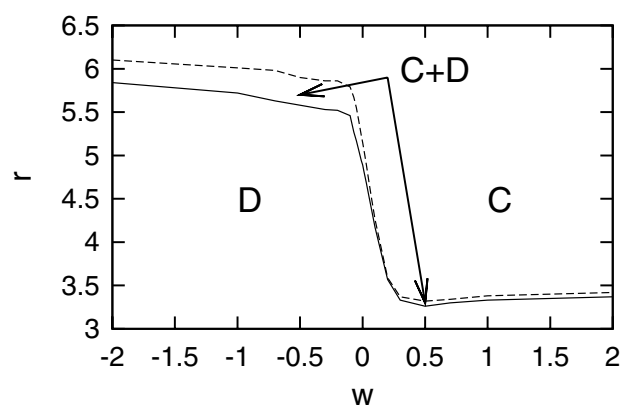

(c)

Fig. 3. Phase diagram for the spatial public goods game with opponent selection biases for (a) (the case of low noise levels) $\kappa=0.1$, (b) (the case of intermediate noise level) $\kappa=0.5$ and (c) (the case of high noise levels) $\kappa=2.0$. Below the solid line cooperators go extinct and above the dashed lines defectors cannot survive. The area between the solid and the dashed line marks the coexistence region.

selection biases can initially help cooperators to avoid interactions with defectors in wellmixed populations and where the undermining effect of negative opponent selection biases on cooperation in structured populations is not overwhelming yet. For this balance of dilemma toughness $r$ and selection bias $w$ cooperators 
can survive, but since cooperators at the fringe of clusters of cooperators preferentially seek out interactions with defectors cooperators find it very hard to dominate.

\subsection{Heterogeneous networks}

The pervious section has demonstrated that biased opponent selection can strongly support cooperation in spatial games. Since most real-world interaction networks have a more heterogeneous topology it is of interest to investigate the influence of network topology on the observed effect. A standard model for heterogeneous networks is the Barabási-Albert model [44], which has frequently been employed in previous studies of evolutionary dilemma games as, e.g., [12-16, 23, 42].

The main effect of network heterogeneity for evolutionary dilemma games is a strong boost for cooperation. The effect results from the superior ability of hub nodes to generate payoff and spread their strategy to adjacent nodes. A cooperator hub can enhance its payoff by spreading the cooperate strategy to its neighborhood this way, but a defector hub would undermine its position by surrounding itself by defectors.

It is important to note that the probabilistic neighbor selection rule of Eq. (1) effectively preserves network heterogeneity for the unbiased situation of $w=0$. Even though every agent selects only one game partner, agents are selected as game partners in proportion to their degrees by other agents, such that an agent with degree $k$ on average takes part in a number of games proportional to $k+1$.

The panels of Fig. 4 summarize simulation data that illustrate the influence of opponent selection biases on cooperation for the case of BA scale-free networks. From panel (a) it is apparent that selecting better opponents $(w>0)$ also improves the stationary numbers of cooperators on scale-free networks. Equally, negative selection biases $w<0$ reduce the chances for the survival of cooperators. The main difference between the situation for regular spatial networks and scale-free networks is an enhanced sensitivity to the bias-parameter in the case of the latter, which results from the strongly uneven abilities of agents to generate payoff on heterogeneous networks.

Due to the pronounced stochasticity in network topology and a strong influence of the initial conditions on stationary fractions of cooperators, simulation data for probabilistic opponent selection on scale-free networks are very noisy and an exact determination of the transitions from dominance of defection to coexistence of defection and cooperation and finally to dominance of cooperation for large $r$ is difficult. Instead of the proper phase diagram, the effect of opponent selection biases on cooperation is well summarized by the dependence of the average of the fraction of cooperators over a suitable interval of dilemma strengths $r$. For convenience I thus define $\left\langle n_{c}\right\rangle_{\mathrm{avg}}=1 / 3.8 \int_{2.5}^{6.3} n_{c}(r) d r$. Numerical estimates for this quantity are shown in panel (b) of Fig. 4. As for the case of spatial networks (cf. Fig. 2) a steep transition in the number of surviving cooperators for $w<0$ and $w>0$ is apparent. 


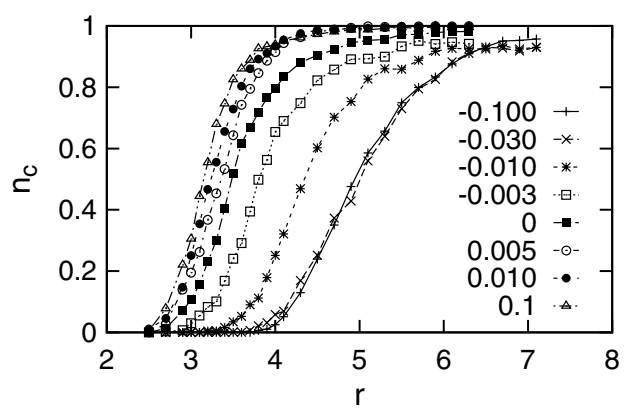

(a)

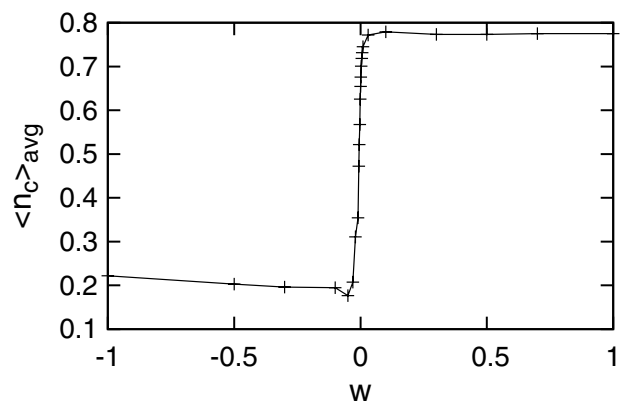

(b)

Fig. 4. (a) Dependence of the average fraction of cooperators $n_{c}$ on $r$ for various opponent selection biases (given in the legend) and BA scale-free network topologies with $\langle k\rangle=4$ and $N=10^{4}$ nodes. (b) Dependence of the average fraction of cooperators averaged over the interval $r \in[2.5,6.3]$ on opponent selection biases $w$. Data points represent averages over 500 independent runs, error bars are about the size of the symbols. The noise level is $\kappa=0.1$.

The figure also highlights the strong sensitivity of cooperation to changes in $w$ for small biases and shows a quick saturation of the effect for biases $|w|>0.1$.

\subsection{Evolution of biases}

So far, the paper has demonstrated that a skewed selection of opponents in PGGs on networks can strongly support the evolution of cooperation. However, it is also of interest whether such opponent selection biases could arise naturally. More pointedly, starting from a randomly mixed population in which some actors prefer playing against more successful opponents and others prefer games with less successful opponents, could a positive selection bias emerge? Further, if such biases emerge, would they be stable against the invasion of new strategies?

To address this question I have designed the following experiment. As before, agents are pure strategists who either cooperate or defect in PGGs. However, additional to this each agent has its individual opponent selection bias $w_{i}$. I assume that these biases are uniformly distributed in the interval $[-1,1]$ in the initial population. 
When selecting opponents for game play (cf. Eq. (1) above), agents select opponents on the basis of their individual preference $w_{i}$. Game play and updating occur is introduced before. The only difference is that now not only strategies, but also the individual traits $w_{i}$ are inherited, based on the rule given in Eq. (3). When inheriting the trait $w_{i}$ a small amount of noise is added, i.e., the preference $w_{j}$ of agent $j$ who inherits $w_{i}$ from agent $i$ is set to $w_{j}=w_{i}+\eta$, where $\eta$ is a small random number drawn uniformly at random from $\eta \in[-0.05,0.05]$.

Additional to the experiments in the previous section, also a small probability of invasions of new strategies into the population is considered. This is implemented in the following way. After strategy adaptation, an agent is replaced by a randomly chosen mutant with a small probability $p_{\text {inv }}$. The mutant has strategy $s=0$ with probability $1 / 2$ and strategy $s=1$ otherwise and has an individual selection bias $w$ drawn uniformly at random from $[-1,1]$.

Opponent selection, game play and strategy adaptation and mutations are then iterated till a quasi-stationary state has been reached. Figure 5 illustrates a typical evolution experiment along these lines. Panel (a) gives the evolution of the fraction of cooperators averaged over 100 trajectories. Starting with a random allocation of strategies, the frequency of cooperators declines at first and recovers when spatial clusters of cooperators have been established and positive opponent selection biases start to emerge. The initial decline is due to the advantage of defection in well-mixed populations. Panel (b) shows the simultaneous emergence of a positive selection bias and illustrates the narrowing down of the variance of biases in the population. Panel (c) complements this by giving snapshots of the distribution of the $w$ parameters in the population at various stages of the evolution.

One first realizes that even though the dilemma setting is close to the smallest extinction threshold achievable for this noise level $\kappa=0.1$ (cf. Fig. 2, close inspection shows that the lowest extinction threshold is $r_{c}=2.85 \pm 0.02$ for $w=0.5$ and $\kappa=0.1$ ) cooperation can actually emerge in this co-evolutionary setting. Further, once achieved cooperation is stable and dominant in the face of a relatively large rate of entry of new mutants into the population (on average 10 new mutants enter the population in each round).

Second, the experiment clearly illustrates that the evolution of cooperation goes hand in hand with the evolution of positive opponent selection biases. This is seen in panel (b). The shift in biases towards $w>0$ is complemented by a narrowing down of the variance of $w$ in the population. By illustrating how the distribution of $w$-parameters in the population gradually shifts towards larger positive values of $w$, panel (c) further corroborates this finding. At the end of the experiment, the bulk of the distribution of the $w$ 's is centered around a mean value of around $w=0.7$. Invasions of randomly selected mutants cause a long tail towards negative values in the distribution.

Cooperation and opponent selection biases cannot only evolve on spatial grids. Very similar experiments for the case of scale-free networks demonstrate that biases will also evolve when the underlying network is heterogeneous. Because typical 


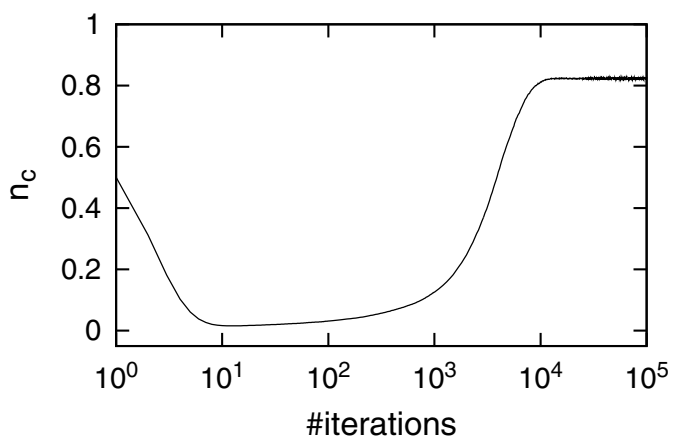

(a)

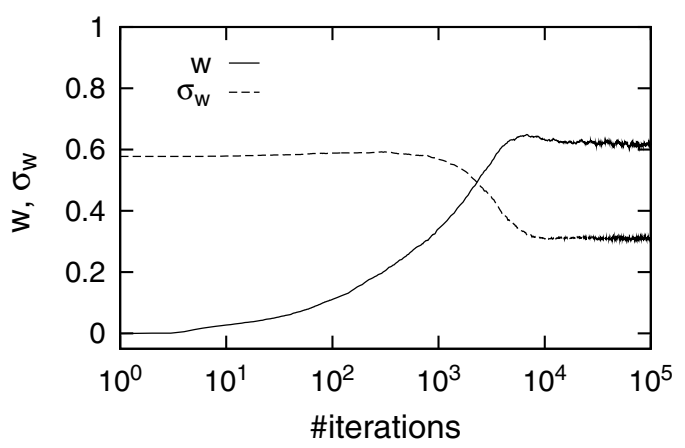

(b)

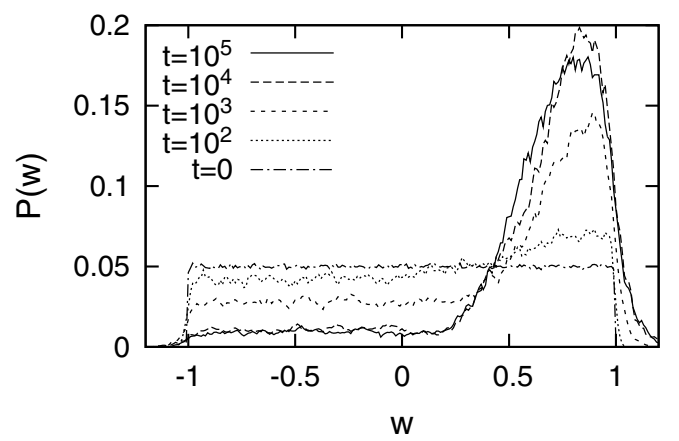

(c)

Fig. 5. Evolution of (a) cooperation and (b) evolution of average biases and variance in the population from random initial distributions of the $w$-parameter. (c) Distribution of the $w$-parameter in the population after $0,10^{2}, 10^{3}, 10^{4}$ and $10^{5}$ timesteps. $b=2.95, \kappa=0.1, p_{\text {inv }}=10^{-3}$. Trajectories represent averages over 100 runs on a $100 \times 100$ square lattice. 
evolutionary paths look very similar to those for spatial grid shown in 5 a detailed discussion is omitted.

\section{Conclusions}

This paper has presented results that extended previous work about opponent selection in the prisoner's dilemma game [32] to PGGs. It is shown that a positive bias to preferentially engage in PGGs with neighbors that previously earned large payoffs can strongly boost cooperation, both on spatial grids as also on scalefree networks. In contrast, biases to engage in games with unsuccessful players undermine cooperation. Opponent selection biases typically shift the onset of cooperation, but also influence the fractions of surviving cooperators in the coexistence phases.

In the second part of the paper, I have also highlighted a mechanism for the emergence of opponent selection biases. Starting with a randomly mixed population in which equal shares of agents have either positive or negative opponent selection biases the strategy of "preferentially playing against successful" opponents will emerge as dominant, if biases are coinherited with game strategies. Importantly, the result is robust in the presence of small rates of invasions of randomly selected mutants.

Altogether, the paper points out a simple mechanism that can explain the presence of cooperation in structured populations, even in very severe dilemma situations.

\section{Acknowledgments}

This research was undertaken on the NCI National Facility in Canberra, Australia, which is supported by the Australian Commonwealth Government.

\section{References}

[1] Weibull, J., Evolutionary Game Theory (MIT University Press, Cambridge, MA, 1996).

[2] Axelrod, R. and Hamilton, W. D., The evolution of cooperation, Science 211 (1981) 1390-1396.

[3] Hamilton, W. D., Genetical evolution of social behaviour I, J. Theor. Biol. 7 (1981) $1-16$.

[4] Axelrod, R. and Dion, D., The further evolution of cooperation, Science 242 (1988) $1385-1390$.

[5] Nowak, M. A. and Sigmund, K., Evolution of indirect reciprocity by image scoring, Nature 393 (1998) 573-577.

[6] Hauert, C., De Monte, S., Hofbauer, J. and Sigmund, K., Volunteering as Red Queen mechanism for cooperation in public goods games, Science 296 (2002) 1129-1132.

[7] Szabó, G. and Hauert, C., Phase transitions and volunteering in spatial public goods games, Phys. Rev. Lett. 89 (2002) 0118101.

[8] Nowak, M. A. and May, R. M., Evolutionary games and spatial chaos, Nature 359 (1992) 826-829. 
[9] Lindgren, K. and Nordahl, M. G., Evolutionary dynamics of spatial games, Phys. D. 75 (1994) 292-309.

[10] Abramson, G. and Kuperman, M., Social games in a social network, Phys. Rev. E 63 (2001) 030901.

[11] Hauert, C. and Doebeli, M., Spatial structure often inhibits the evolution of cooperation in the snowdrift game, Nature 428 (2004) 643-646.

[12] Santos, F. C., Pacheco, J. M. and Lenaerts, T., Evolutionary dynamics of social dilemmas in structured heterogenous populations, Proc. Natl. Acad. Sci. USA 103 (2006) 3490-3494.

[13] Santos, F. C., Rodrigues, J. F. and Pacheco, J. M., Graph topology plays a determinant role in the evolution of cooperation, Proc. R. Soc. B. 273 (2006) 51-55.

[14] Masuda, N., Participation costs dismiss the advantage of heterogenous networks in evolution of cooperation, Proc. R. Soc. B. 274 (2007) 1815-1821.

[15] Santos, F. C., Rodrigues, J. F. and Pacheco, J. M., Social diversity promotes the emergence of cooperation in public goods games, Nature 454 (2008) 213-215.

[16] Tanimoto, J. and Yamauchi, A., Does "game participation cost" affect the advantage of heterogenous networks for evolving cooperation? Physica A 389 (2010) 2284-2289.

[17] Szabó, G. and Fath, G., Evolutionary games on graphs, Phys. Rep. 446 (2007) 97-216.

[18] Albert, R. and Barabási, A.-L., Statistical mechanics of complex networks, Rev. Mod. Phys. 74 (2002) 47-97.

[19] Newman, M. E. J., The structure and function of complex networks, SIAM Rev. 45 (2003) 167-256.

[20] Boccaletti, S., Latora, V., Moreno, Y., Chavez, M. and Hwang, D.-U., Complex networks: Structure and dynamics, Phys. Rep. 424 (2006) 175-308.

[21] Perc, M., Coherence resonance in a spatial prisoner's dilemma game, New J. Phys. 8 (2006) 022.

[22] Perc, M. and Szolnoki, A., Social diversity and promotion of cooperation in the spatial prisoner's dilemma game, Phys. Rev. E 77 (2008) 011904.

[23] Brede, M., The evolution of cooperation on correlated payoff landscapes, Artif. Life 17 (2011) 365-373.

[24] Szolnoki, A. and Szabó, G., Cooperation enhanced by inhomogeneous activity of teaching for evolutionary prisoner's dilemma game, EPL 77 (2007) 30004.

[25] Chadefaux, T. and Helbing, D., How wealth accumultion can promote cooperation, PLoS ONE 5 (2010) e13471.

[26] Perc, M., Success-driven distribution of public goods promotes cooperation but preserves defection, Phys. Rev. E 84 (2011) 037102.

[27] Wang, Z. and Perc, M., Aspiring to the fittest and promotion of cooperation in the prisoner's dilemma game, Phys. Rev. E 82 (2010) 002115.

[28] Perc, M. and Wang, Z., Heterogenous aspirations promote cooperation in the prisoner's dilemma game, PLoS ONE 5 (2010) e15117.

[29] Zhang, H., Small, M., Yang, H. and Wang, B., Adjusting learning motivation to promote cooperation, Physica A 389 (2010) 4734-4739.

[30] Shi, D.-M., Yang, H.-X., Hu, M.-B., Du, W.-B., Wang, B.-H. and Cao, X.-B., Preferential selection promotes cooperation in a spatial public goods game, Physica A $\mathbf{3 8 8}$ (2009) 4646-4650.

[31] Zhang, H., Liu, R., Wang, Z., Yang, H. and Wang, B., Aspiration-induced reconnection n spatial public goods game, EPL 94 (2011) 18006.

[32] Brede, M., Playing against the fittest: A simple strategy that promotes the emergence of cooperation, EPL 94 (2011) 30003. 
[33] Zimmermann, M. G., Eguíluz, V. M. and San Miguel, M., Coevolution of dynamical states and interactions in dynamic networks, Phys. Rev. E 69 (2004) 065102(R).

[34] Zimmermann, M. G. and Eguíluz, V. M., Cooperation, social networks, and the emergence of leadership in a prisoner's dilemma with adaptive local interactions, Phys. Rev. E $\mathbf{7 2}$ (2005) 056118.

[35] Pacheco, J. M., Traulsen, A. and Nowak, M. A., Coevolution of strategy and structure in complex networks with dynamical linking, Phys. Rev. Lett. 97 (2006) 258103.

[36] Wu, T., Fu, F. and Wang, L., Partner selections in public goods games with constant group size, Phys. Rev. E 80 (2009) 026121.

[37] Szolnoki, A. and Perc, M., Resolving social dilemmas on evolving random networks, EPL 86 (2009) 30007.

[38] Van Segbroeck, S., Santos, F. C., Lenaerts, T. and Pacheco, J. M., Reacting differently to adverse ties promotes cooperation in social networks, Phys. Rev. Lett. 102 (2009) 058105.

[39] Tanimoto, J., Promotion of cooperation through co-evolution of networks and strategy in a $2 \times 2$ game, Physica A 388 (2009) 953-960.

[40] Cao, L., Ohtsuki, H., Wang, B. and Aihara, K., Evolution of cooperation on adaptively weighted networks, J. Theor. Biol. 272 (2011) 8-15.

[41] Perc, M. and Szolnoki, A., Coevolutionary games - A mini review, BioSystems 99 (2010) 109-125.

[42] Poncela, J., Gómez-Gardenes, J. and Moreno, Y., Cooperation in scale-free networks with limited associative capacities, Phys. Rev. E 83 (2011) 057101.

[43] Szabó, G. and Töke, C., Evolutionary prisoner's dilemma game on a square lattice, Phys. Rev. E 58 (1998) 69-73.

[44] Barabási, A.-L. and Albert, R., Emergence of scaling in random networks, Science 286 (1999) 509-512. 